\title{
COMMENT
}

\section{Neurobiological consequences of racial disparities and environmental risks: a critical gap in understanding psychiatric disorders}

\author{
Nathaniel G. Harnett ${ }^{1,2}$ \\ Neuropsychopharmacology (2020) 45:1247-1250; https://doi.org/10.1038/s41386-020-0681-4
}

Racial groups within the United States show marked differences in prevalence rates for numerous psychiatric disorders. Generally, minoritized racial groups tend to show lower prevalence of mental illness compared with White-American groups, although some disorders are more commonly diagnosed in minoritized groups (e.g., schizophrenia) [1, 2]. Increased interest over the last decade has been placed on the neural substrates that mediate the development and expression of these psychiatric disorders. However, while our understanding of the neurobiological substrates of psychiatric disorders has grown, almost no attention has been paid to whether or how the neural substrates of these disorders may vary between racial groups. Importantly, minoritized racial groups are differentially exposed to environmental risk factors (e.g., early life adversity) that are known to play a role in a variety of psychiatric disorders $[3,4]$. There is significant potential for racial disparities in environmental risk factors to moderate the relationship between neurobiology and psychiatric disorder development that is currently unexplored.

Race as a concept incorporates complex social and historical dynamics that contribute to significant variability in environmental exposures and lived experiences between racial groups [5]. Failure to properly investigate such between-group environmental variability may lead to the unfortunate possibility of developing exclusionary disease models that will (a) not generalize to the wider population, (b) facilitate treatment inequality and inefficacy, and (c) undermine our fundamental understanding of the pathobiology of psychiatric disorders. Therefore, it is critical that we begin to understand the neurobiological consequences of racial disparities in environmental risk factors and the potential effects on group differences in the prevalence of psychiatric disorders.

\section{ENVIRONMENTAL DISPARITIES AND THEIR POTENTIAL NEUROBIOLOGICAL CONSEQUENCES}

Environmental factors have strong direct and indirect effects on both neurobiology and psychiatric disorders. Early life events such as exposure to toxins, childhood trauma, maternal stress, immune system activation, and other risk factors show clear effects on the neural substrates of emotion and stress regulation. In particular, prefrontal cortex-hippocampus-amygdala circuity, important for regulation of the emotional response to threat $[6,7]$, and the hypothalamic pituitary adrenal (HPA) axis, a key mediator of the stress response [8], are impacted by environmental risk factors and these substrates are in turn implicated in several psychiatric disorders $[9,10]$. The extant literature presents a clear relationship between environmental exposures, neural circuitry, and increased risk for psychiatric disorders.

Although environmental risk factors can affect any individual, there are considerable differences in exposures to environmental risks between racial groups. Minoritized racial groups are-in general-more often exposed to adversity, such as childhood maltreatment or violence [11]. Minoritized racial groups also have lower family income and wealth, and often live in areas with greater disadvantage compared with non-minoritized racial groups [12]. Recent research further suggests minoritized racial groups have increased exposure to toxins throughout life which can have potentially deleterious effects on physical and mental health [13]. There are also significant differences in the general lived experiences of minoritized racial groups, such that these groups are exposed to highly impactful race-related stressors. Racial discrimination, both at personal and institutional levels, can be psychologically damaging and further alter the neurobiology of psychiatric disorders [14]. Overt and covert biases and behaviors between racial groups can also contribute to race-related stress that represent a significant aspect of between-group variability in the environment. Further, it should be noted that these racerelated stressors are not independent of other environmental risk factors (e.g., poverty and violence) such that these risk factors interact with, and exacerbate, health inequities [15]. Considering all these factors together, there are significant differences in environmental risk for psychiatric disorders between racial groups.

Despite evidence that environmental risk factors have measurable effects on the neurobiological substrates of psychiatric disorders, and the observable between-group variability in these risk factors, there is limited data on the neurobiological consequences of such racial disparities in environmental stressors. Prior work has observed differences in Black-American and WhiteAmerican participants' gray matter structure, particularly within the orbitofrontal cortex [16]. Although no information on environmental risk factors were available in that report, the findings suggest at the very least there may be race-related differences in neurobiology, particularly in regions important for regulating emotional responses. The observed differences are likely due to multifactorial causes which may include potential differences in exposures to environmental stressors. A recent report found that Black-American participants-who had greater levels of exposure to violence, lower family incomes, and greater

${ }^{1}$ Division of Depression and Anxiety, McLean Hospital, Belmont, MA, USA and ${ }^{2}$ Department of Psychiatry, Harvard Medical School, Boston, MA, USA

Correspondence: Nathaniel G. Harnett (nharnett@mclean.harvard.edu)

Received: 10 January 2020 Revised: 11 April 2020 Accepted: 15 April 2020

Published online: 24 April 2020 
neighborhood disadvantage throughout life-showed blunted neural and behavioral reactivity to threat compared to WhiteAmerican participants [17]. Given responses to threat are often different in individuals with mood disorders (e.g., depression, anxiety, and PTSD), one may speculate that these race-related differences are related to potential resiliency, or susceptibility, mechanisms for psychiatric disorders. Another study also observed differences in brain structure and neurocognitive domains of psychiatric symptoms between racial groups, particularly Black and White-American groups, and these brain and cognitive variables interacted with environmental factors such as trauma and socioeconomic status [18]. These data further highlight the complex interactions between race, environment, and the neurobiological substrates of psychiatric disorders. However, it remains uncertain whether these patterns may be observed in other minoritized racial groups that show differences in mental illness prevalence (e.g., Hispanic/Latinx, Indigenous/Native-American, and other minoritized populations) [1, 19]; further research is needed to more fully and carefully disentangle the relationship between racial disparities in environment stressors and neurobiology of psychiatric disorders.

\section{IMPROVING OUR UNDERSTANDING OF THE NEURAL CONSEQUENCES OF DISPARITIES IN ENVIRONMENTAL RISK}

Research on the impact of racial disparities in environmental risk factors on the brain is needed for a fuller understanding of the development and maintenance of psychiatric disorders. Several strategies would enable a clearer understanding of such impacts and shine new light on interactions among the environment, the brain, and psychiatric disorders. One important strategy is the use of preclinical (e.g., rodent) models to study the impact of environmental variables on specific cellular and molecular pathways already known to play a role in psychiatric disorder development. Specific and targeted experiments on environmental deprivation/enrichment could pinpoint behavioral, neuronal, and genetic consequences related to resilience or susceptibility to psychiatric disorders [20]. For example, combining environmental deprivation (as a proxy for relative disadvantage) with well-established models of mood disorders (e.g., chronic stress [21]) may provide insight into interactions among different types of environmental factors, as well as accumulating stressors, on key cellular and molecular dynamics of neural circuits involved in emotion and stress regulation. Another key benefit of using animal models is the potential to test for transgenerational effects that may be important for understanding the neurobiological consequences of racial disparities in environmental risk $[22,23]$. As an example, recent research has demonstrated transgenerational epigenetic modulation of FKBP5, a major regulator of HPA axis activity, in humans [24, 25]. Animal models would thus allow for the study of mechanisms of these effects and facilitate our understanding of the neural effects of environmental differences across multiple generations. However, it should be acknowledged that complex race-related issues in humans are challenging to recapitulate in animal models. For example, racial discrimination and race-related traumas (e.g., segregation or racial violence) involve domains that are not easily modeled in rodents. Thus while animal models will be invaluable for understanding neurobiological aspects of environmental risk factors, their usefulness for fully understanding complex and interacting race-related differences in environmental risk has inherent limitations.

Human neuroimaging and neurogenetic research is also needed to better understand the neurobiological consequences of racial disparities in environmental risk factors. Neuroimaging and neurogenetic research samples tend to be nonrepresentative of racial diversity within the United States, and few studies have investigated the relationship between environmental variables, race, and neural processes $[16-18,26]$. The limited representation of minoritized racial groups in research samples thus limits generalizability of findings. Although some studies may include minoritized racial groups, a common strategy to address potential race-related effects is to include race as a covariate in statistical tests to assess the unique relationship between an environmental risk factor and the brain. However, considering the racial disparities in environmental risk (described above), it is necessary to consider not just the unique relationship between environment and the brain but also potential interactions with race. Understanding between-group differences in neural processes in conjunction with racial disparities in environmental risk factors is critical to fully characterize the role of environmental risk factors on the neurobiology of psychiatric disorders. Of note, recent largescale collaborative studies such as the ABCD study [27], ENIGMA consortia [28], and PGC [29] offer a novel chance to better consider race-related effects through more representative data collection and cross-study data pooling. Further research is needed in humans to identify the relationship between the neurobiological of impact racial disparities in environmental risk factors, and their influence on race-related differences in psychiatric disorders.

\section{BARRIERS}

Despite the need for increased overall research, there are significant systemic barriers against research into environmental racial disparities and their consequences that must first be addressed. For one, there is a general lack of funding for research questions related to racial disparities in environment compared to other topics [30]. Failure to secure funding for research topics often means that these research questions go unanswered and may lead to a loss of knowledge about an important public health topic. Importantly, health disparities research is more often pursued by researchers from minoritized racial groups [30]. The lack of funding support for these topics may thus result in the further consequence of attrition of researchers from minoritized racial groups. The attrition of these individuals is a major detriment to research as these individuals offer a diversity of viewpoints and ideas that contribute to scientific advancement. At a time where research funding is already of concern to many, it is difficult to determine the best course for rectifying this particular funding gap. One solution proposed by the National Institutes of Health (NIH) has been to convene special study sections and funding opportunities to address these research and funding gaps. Greater support for health disparities research will lead to better understanding the neurobiological consequences of disparities in environmental risk factors for psychiatric disorders, as well as better understanding the neurobiology of behavior more broadly.

A second barrier to progress in this area concerns the potential for discomfort that discussions of race can elicit, particularly in light of past controversies surrounding the psychological study of race-related differences [31]. Specifically, there may be worry over how findings of race-related differences may be misinterpreted within the wider public (e.g., that these may be viewed as innate or immutable differences). Findings of race-related differences in brain function can be subject to unwarranted reverse causal inference. For example, some individuals may read about a potential finding of race-related differences in "brain function" and overinterpret the data in a way that happens to reinforce a preconceived belief. A solution to mitigate this potential misunderstanding is to appropriately contextualize any finding of neurobiological differences between racial groups around the specific cognitive process studied and to make explicit note of any measured racial disparities in environmental risk factors. For example, a neuroimaging report of "reduced brain activation" within a racial group would be inappropriate. Instead, findings should be reported for a specific (set of) brain region(s) for a particular task or contrast (e.g., amygdala reactivity to threat cues). 
Further, any race-related differences in environmental factors (e.g., income, violence, etc.) should also be reported to contextualize findings of race-related differences.

The potential for misinterpretation highlights that precise and objective language are crucial for investigation of race-related differences in the neurobiology of psychiatric disorders. Although "race" was conceptualized at the outset of the present paper, it is a term used in several different ways in contemporary discourse. The multiplicity of meanings for the word "race" can thus lead to unintentional ambiguity and potential misinterpretation of scientific results. It may be advantageous for the field to adopt a set of standardized, accurate, and inclusive language guidelines to better facilitate the design of experiments, assessment of variables, and interpretations of race-related research. Given the potential for miscommunication, it is critically important for researchers to take great care during planning, execution, and dissemination of findings related to race-related differences to avoid potentially reinforcing negative stereotypes or perpetrating harmful, unscientific ideas. However, only by studying racial disparities in environmental risk factors, and improving our understanding of their impacts on the brain, can we begin to unravel the mechanisms that underlie differences in prevalence of psychiatric disorders between racial groups.

Finally, cross-cultural differences regarding health care and psychiatric symptoms are another potential barrier to research. Environmental factors (e.g., neighborhood disadvantage) can result in reduced access to psychiatric care and may become barriers that lead to reduced treatment seeking behaviors. Further, given negative environmental factors, minoritized racial groups may engage in coping behaviors that mask the typical presentation of psychiatric symptoms which may facilitate inaccurate diagnoses or misattribution of symptom presentations. Although these factors may impact reported rates of psychiatric disorders directly, these important aspects may also influence research on the neurobiology of psychiatric disorders such that they mask potential race-related findings. Understanding race-related differences in psychiatric access, presentation, and diagnosis are also important areas to consider for research on impact of race disparities in environment on neural circuitry.

\section{CONCLUSIONS}

The neurobiological consequences of racial disparities in environmental risk factors are currently not well characterized. Despite the evidence that environmental factors are robustly able to shape both the brain and development of psychiatric disorders, it is yet unclear how these relationships may manifest in groups differentially exposed to environmental, chemical, physical, psychological, and social stressors. A better understanding of these effects has important implications for our conceptualization of the neurobiology of psychiatric disorders. There are likely important moderating and interacting environmental factors that add significant complexity to the relationship between the brain and the environment. Understanding the interplay of the environment and the brain, particularly in different racial groups that have variable and complex levels of exposure to environmental stress, would provide important insight into why some mental illnesses have a lower prevalence in minoritized racial groups whereas others have higher prevalence. Race-related differences in the neurobiology of psychiatric disorders are likely not simple and different environmental and biological pathways toward risk and resilience must be considered carefully and empirically. It will be a difficult endeavor to disentangle the impact of racial disparities in environmental risk factors on the brain. However, doing so is necessary for the development of generalizable neurobiological models of psychiatric disorders, diminishing the prevalence of mental illness, and improving health for all individuals.
FUNDING AND DISCLOSURE

$\mathrm{NGH}$ is currently supported by NIH grant K00MH119603. The author has no financial disclosures.

\section{ACKNOWLEDGEMENTS}

The author would like to thank Marilyn Thomas, Sikoya Ashburn, Javier How, Kerry Ressler, and William Carlezon for helpful and insightful comments in drafting this paper.

\section{ADDITIONAL INFORMATION}

Publisher's note Springer Nature remains neutral with regard to jurisdictional claims in published maps and institutional affiliations.

\section{REFERENCES}

1. Substance Abuse and Mental Health Services Administration. Key substance use and mental health indicators in the United States: results from the 2017 National Survey on Drug Use and Health (HHS Publication No. SMA 185068, NSUDH Series H-53). Rockville, MD: Center for Behavioral Health Statistics and Quality, Substance Abuse and Mental Health Services Administration; 2018.

2. Coleman KJ, Stewart C, Waitzfelder BE, et al. Racial-ethnic differences in psychiatric diagnoses and treatment across 11 health care systems in the mental health research network. Psychiatr Serv. 2016;67:749-57.

3. Sacks V, Murphey D. The prevalence of adverse childhood experiences, nationally, by state, and by race or ethnicity. Bethesda, Maryland: Child Trends; 2018. https://www.childtrends.org/publications/prevalence-adverse-childhoodexperiences-nationally-state-race-ethnicity/.

4. McLaughlin KA, Green JG, Gruber MJ, Sampson NA, Zaslavsky AM, Kessler RC. Childhood adversities and first onset of psychiatric disorders in a national sample of US adolescents. Arch Gen Psychiatry. 2012;69:1151-60.

5. Ford $\mathrm{CL}$, Airhihenbuwa $\mathrm{CO}$. The public health critical race methodology: praxis for antiracism research. Soc Sci Med. 2010;71:1390-8.

6. Goodman AM, Harnett NG, Knight DC. Pavlovian conditioned diminution of the neurobehavioral response to threat. Neurosci Biobehav Rev. 2018;84:218-24.

7. Carlezon WA, Kim W, Missig G, Finger BC, Landino SM, Alexander AJ, et al. Maternal and early postnatal immune activation produce sex-specific effects on autism-like behaviors and neuroimmune function in mice. Sci Rep. 2019;9:16928.

8. Smith SM, Vale WW. The role of the hypothalamic-pituitary-adrenal axis in neuroendocrine responses to stress. Dialogues Clin Neurosci. 2006;8:383-95.

9. Mehta D, Binder EB. Genex environment vulnerability factors for PTSD: the HPAaxis. Neuropharmacology. 2012;62:654-62.

10. Herringa RJ, Birn RM, Ruttle PL, Burghy CA, Stodola DE, Davidson RJ, et al. Childhood maltreatment is associated with altered fear circuitry and increased internalizing symptoms by late adolescence. Proc Natl Acad Sci USA. 2013;110:19119-24.

11. Slopen N, Shonkoff JP, Albert MA, Yoshikawa H, Jacobs A, Stoltz R, et al. Racial disparities in child adversity in the US: Interactions with family immigration history and income. Am J Prev Med. 2016;50:47-56.

12. Williams DR, Collins C. Racial residential segregation: a fundamental cause of racial disparities in health. Public Health Rep. 2001;116:404-16.

13. Tessum CW, Apte JS, Goodkind AL, Muller NZ, Mullins KA, Paolella DA, et al. Inequity in consumption of goods and services adds to racial-ethnic disparities in air pollution exposure. Proc Natl Acad Sci USA. 2019;116:6001-6.

14. Clark US, Miller ER, Hegde RR. Experiences of discrimination are associated with greater resting amygdala activity and functional connectivity. Biol Psychiatry Cogn Neurosci Neuroimaging. 2018;3:367-78.

15. Bailey ZD, Krieger N, Agénor M, Graves J, Linos N, Bassett MT. Structural racism and health inequities in the USA: evidence and interventions. Lancet. 2017;389:1453-63.

16. Isamah N, Faison W, Payne ME, MacFall J, Steffens DC, Beyer JL, et al. Variability in frontotemporal brain structure: the importance of recruitment of African Americans in neuroscience research. PloS ONE. 2010;5:e13642.

17. Harnett NG, Wheelock MD, Wood KH, Goodman AM, Mrug S, Elliott MN, et al. Negative life experiences contribute to racial differences in the neural response to threat. Neurolmage. 2019;202:116086.

18. Gur RE, Moore TM, Rosen AF, Barzilay R, Roalf DR, Calkins ME, et al. Burden of environmental adversity associated with psychopathology, maturation, and brain behavior parameters in youths. JAMA Psychiatry. 2019;76:966-75. 
Neurobiological consequences of racial disparities and environmental...

NG Harnett

1250

19. Salinas C Jr, Lozano A. Mapping and recontextualizing the evolution of the term Latinx: an environmental scanning in higher education. J Lat Educ. 2019;18:302-15.

20. Francis DD, Diorio J, Plotsky PM, Meaney MJ. Environmental enrichment reverses the effects of maternal separation on stress reactivity. J Neurosci. 2002;22:7840-3.

21. Akil H, Gordon J, Hen R, Javitch J, Mayberg H, McEwen B, et al. Treatment resistant depression: a multi-scale, systems biology approach. Neurosci Biobehav Rev. 2018;84:272-88.

22. Gapp K, Bohacek J, Grossmann J, Brunner AM, Manuella F, Nanni P, et al. Potential of environmental enrichment to prevent transgenerational effects of paternal trauma. Neuropsychopharmacology. 2016;41:2749.

23. Bale TL. Epigenetic and transgenerational reprogramming of brain development. Nat Rev Neurosci. 2015;16:332-44.

24. Binder EB. The role of FKBP5, a co-chaperone of the glucocorticoid receptor in the pathogenesis and therapy of affective and anxiety disorders. Psychoneuroendocrinology. 2009;34:S186-95.

25. Yehuda R, Daskalakis NP, Bierer LM, Bader HN, Klengel T, Holsboer F, et al. Holocaust exposure induced intergenerational effects on FKBP5 methylation. Biol Psychiatry. 2016;80:372-80.
26. LeWinn KZ, Sheridan MA, Keyes KM, Hamilton A, McLaughlin KA. Sample composition alters associations between age and brain structure. Nat Commun. 2017;8:1-14

27. Garavan H, Bartsch H, Conway K, Decastro A, Goldstein RZ, Heeringa S, et al. Recruiting the $A B C D$ sample: design considerations and procedures. Developmental Cogn Neurosci. 2018;32:16-22.

28. Thompson PM, Stein JL, Medland SE, Hibar DP, Vasquez AA, Renteria ME, et al. The ENIGMA Consortium: large-scale collaborative analyses of neuroimaging and genetic data. Brain Imaging Behav. 2014;8:153-82.

29. Logue MW, Amstadter AB, Baker DG, Duncan L, Koenen KC, Liberzon I, et al. The Psychiatric Genomics Consortium Posttraumatic Stress Disorder Workgroup: posttraumatic stress disorder enters the age of large-scale genomic collaboration. Neuropsychopharmacology. 2015;40:2287-97.

30. Hoppe TA, Litovitz A, Willis KA, Meseroll RA, Perkins MJ, Hutchins BI, et al. Topic choice contributes to the lower rate of $\mathrm{NIH}$ awards to African-American/black scientists. Sci Adv. 2019;5:eaaw7238.

31. Richards G. Race, racism and psychology: towards a reflexive history. New York, NY: Routledge; 2012. 